\title{
La motivación en la música como eje de la formación profesional
}

\section{Motivation in music as the focus of professional training}

\author{
JUAN-CARVAJAL, Mara Lioba*†, JUAN-CARVAJAL, Dargen Tania y VDOVINA, María \\ Universidad Autónoma de Zacatecas, México \\ Universidad de las Artes, Cuba \\ Universidad Autónoma de Zacatecas, México \\ ID $1^{\text {er }}$ Autor: Mara Lioba, Juan-Carvajal / ORC ID: 0000-0001-6968-3813, Researcher ID Thomson: P-7756-2016, CVU \\ CONACYT ID: 216443
}

ID $1^{\text {er }}$ Coautor: Dargen Tania, Juan-Carvajal / ORC ID: 0000-0002-8281-8169, Researcher ID Thomson: V-7816-2019

ID $2^{\text {do }}$ Coautor: María, Vdovina / ORC ID: 0000-0001-6656-0789, Researcher ID Thomson: S-7917-2018

DOI: $10.35429 / J U M .2020 .11 .4 .1 .9$

Recibido 12 de Marzo, 2020; Aceptado 30 de Junio, 2020

\section{Resumen}

En algunos países la enseñanza de la música está presente en los planes de estudio desde edades tempranas, ello provee a los escolares una cultura general y, en ciertos casos, los estimula para continuar los estudios en los niveles superiores. La decisión de profesionalizarse como músico, implica un gran sacrificio para el joven que, normalmente, empieza desde pequeño y cuenta con el apoyo familiar, pero que, hacia la adolescencia, requiere diversas estrategias motivadoras que lo estimulen a terminar los estudios a nivel licenciatura. La oferta de carreras relativas a la música es diversa en gran parte de los estados mexicanos; sin embargo, en muchos casos, la música no es el eje principal en su educación. Ello conllevó a esta reflexión que tiene por objetivo recapitular sobre aquellos aspectos que motivan a los estudiantes a formarse como músicos profesionales.

Motivación, Música, Motivación hacia el estudio

\begin{abstract}
In some countries, music teaching is present in the study plans since an early age for kids. This provides the schoolchildren with general culture and, in certain cases, encourages them to continue their musical studies at higher levels of education. The decision to major as a musician, though, implies a great sacrifice for a teen who, in ideal terms, would start from a young age and would have family support, but towards adolescence, requires various motivational strategies that encourage him to finish his studies at a bachelor's level. The career choice related to music education is diverse in most of the Mexican states; however, in many cases, music is not the main focus of the education plans. These considerations led to this reflection, which aims to recapitulate and examine the aspects that motivate students to train as professional musicians.
\end{abstract}

Motivation, Music, Motivation towards study

Citación: JUAN-CARVAJAL, Mara Lioba, JUAN-CARVAJAL, Dargen Tania y VDOVINA, María. La motivación en la música como eje de la formación profesional. Revista de Gestión Universitaria. 2020. 4-11: 1-9

\footnotetext{
* Correspondencia del Autor (Correo electrónico: maralioba@ hotmail.com)

$\dagger$ Investigador contribuyendo como primer autor.
} 


\section{Introducción}

En la literatura universal podemos encontrar un amplio espectro de referentes relacionados con el impacto de la música en el desarrollo del ser humano. En los períodos reconocidos para ilustrar la evolución del hombre, aparecen las artes y dentro de ellas la música, como un componente latente del progreso social; es claro que no en todas las épocas y regiones su comportamiento ha sido análogo, y en unos referentes, se distingue el papel de ocio que mayoritariamente se le ha otorgado. Quizás por esta razón, en ciertas regiones el estudio de la música no es el eje fundamental en la formación profesional, sino que se toma como complemento a otra carrera que se supone "de mayor importancia social". Sin embargo, existe un sinnúmero de actividades en las que la presencia del arte musical adquiere un rol revelador, sobre todo en lo que aporta a la calidad de vida, a la expresión de los sentimientos, las emociones, la comunicación; como apoyo a actividades laborales, comunitarias, sociales y a la identidad como baluarte, entre otros aspectos. Tal vez por ello el famoso slogan de que la música es un lenguaje universal.

A propósito de esta temática, Lorenzo (2020) enuncia su alta valoración regeneradora al manifestarse en ámbitos con impacto socioeconómico: protección y reforma de menores; instituciones penitenciarias; residencias para personas de la tercera edad; mujeres maltratadas, madres adolescentes; centros de tratamientos de drogodependencias; centros hospitalarios (especialmente de larga estancia); discapacitados físicos y psíquicos. "La transformación social mediante la música es posible gracias a las iniciativas fundamentadas principalmente por la educación musical, el arte y la cultura que promueve la paz..." (Rueda, 2020, p.29). Miranda, Hazard, y Miranda (2017), declaran que la música constituye una herramienta terapéutica en medicina y destacan su potencial en diversos tratamientos neurológicos, así como en los casos de dolor y ansiedad en enfermedades somáticas y cáncer.

La antigüedad del uso terapéutico de la música (lo que hoy se conoce como musicoterapia) y su importancia sociocultural quedó recogida en el Diccionario de música, mitología, magia y religión de González-Cobo (2014):
La música, empleada como medicina y remedio de las enfermedades físicas y psíquicas, cuenta con un lejano origen en el tiempo. El fenómeno del tratamiento y curación mediante los sonidos, muy ligado a las ceremonias de magia, fue común a las más diversas civilizaciones, en las que los ritos de sanación estaban estrechamente vinculados a una visión cosmológica del mundo interpretada a través del cuerpo. Sabemos que en Egipto la música era empleada tanto para combatir las plagas como para apaciguar el ánimo y regular y crear la compostura del cuerpo físico o jat, que debía permanecer concorde con el ba y el ka a fin de que todo fluyera apaciblemente. De manera semejante acaeció en la cultura mesopotámica, donde la música fue comúnmente utilizada en los actos de purificación -no sólo litúrgicos-, durante los cuales la sonoridad tenía el cometido de «limpiar» al enfermo. Los sumerios, al igual que los hititas, fueron proclives a esta clase de rituales, en los que intervenía un canto que no debía de distar mucho del que acompañaba, dentro del templo, al sacerdote purificador llamado ishib o sanga. Si se tiene en cuenta que para los sumerios, como después sucedió entre los babilonios, la enfermedad acostumbraba a ser la consecuencia de una posición demoníaca (diablo), es presumible el valor conferido a la música y la danza, habida cuenta de su capacidad como elemento de exorcismo. (pp. 532-533)

El arte musical ha tenido a través de los tiempos una doble funcionalidad respecto a su accionar con el humano; ha aportado al equilibrio cuerpo-mente, salud y paz, y también ha sido un instrumento de comunicación, una manera de exteriorizar experiencias, vivencias, y cosmovisiones; de ahí ese vínculo con la mitología, la astrología, la magia, las religiones y el mundo espiritual. Toda la música compuesta, aún en los tiempos actuales, ha sido inspirada por ciertos elementos que los compositores perciben en su interior, pero que, ocasionalmente, se sienten incapaces de externar, a no ser por lo que ella dice en sí misma cuando es interpretada, cuando es escuchada. De la misma manera, cuando se interpreta la música se requiere de un conocimiento amplio del contexto de su creación y su posible significación, sin embargo, cada interpretación es única e irrepetible aún cuando se trate del mismo ejecutante. 
Esta esencia misteriosa la convierte en una expresión insuperable, fugaz en el tiempo, vívida e inmaterial, y quizás no siempre entendida en la cotidianidad de una vida práctica y razonable, por lo que, en las sociedades actuales, aunque todos la disfruten de una u otra forma, algunos no la consideran una "carrera real", una opción de trabajo o un modo de vida responsable y con perspectiva de progreso.

Pese a lo anterior, en el ámbito educativo se elogia la presencia de la música como un componente esencial en la formación de valores; en este sentido se distingue la identidad, la cooperación y la aceptación de la diversidad teniendo en cuenta los rasgos personales, el establecimiento de nexos sociales y la capacidad del alumno de alcanzar las metas propuestas (Conejo, 2012). También es importante en el desarrollo del lenguaje, de competencias, y en el proceso comunicativo, “... algunas investigaciones explican cómo la melodía y el ritmo facilitan el aprendizaje y la memorización" (Piñeros, 2016, p.66). En contraste con ello, en varias instituciones docentes donde la enseñanza de las artes era obligatoria, comienza un proceso de reestructuración en el que se elimina de los planes formativos.

El resultado de esta menor presencia de la música en el sistema escolar es una menor cantidad de recursos disponibles tanto en dinero como en tiempo, y una reducción de la música también de los planes de estudio, mientras que la vida del alumnado fuera de las aulas está rodeada de música. La gente joven consume y produce música a través de redes sociales y contextos informales, siendo una parte importante de las identidades juveniles, al tiempo que permite desarrollar aspectos psicomotrices, socioafectivos y cognitivos del ser humano. (Aróstegui, Louro, y Lima, 2015, p.25)

En muchas escuelas de México la educación artística inicia desde los niveles primarios, en ella se desarrollan programas acordes con las políticas educacionales del país.
La música, como parte de la Educación Artística, debe enseñarse junto con artes visuales, teatro y danza. En respuesta a la Reforma Integral de la Educación Básica (Subsecretaría de Educación Básica, 2008), los planes de estudio para la Educación Artística proponen un enfoque metodológico llamado en «ensamble artístico». En un enfoque integrador, éste toma la forma de proyectos que proporcionan a los estudiantes la oportunidad de aplicar, reflexionar e integrar los conocimientos adquiridos a través de las cuatro áreas artísticas. Cada área es abordada a través de seis ejes de aprendizaje: a) percepción sensorial; b) contemplación; c) expresión; d) apreciación; e) contextualización; f) creación. Por medio de estos ejes de aprendizaje, los estudiantes deben cumplir con las competencias en materia de percepción estética, interpretación abstracta y comunicación creativa. (González-Moreno, 2013, p.33)

Es decir, la educación artística general es ahora un proceso de enseñanza-aprendizaje conjunto y armónico, impacta en el desarrollo del niño y el adolescente de manera integral, y considera las líneas educativas propuestas a través de ejes que favorecen cambios en las esferas cognitiva y afectiva. Por consiguiente, los niños que desean iniciar estudios formales de música (que en algunos instrumentos como el piano o el violín se empieza a partir de los 7 u 8 años) además de ganar una cultura general mediante los elementos implícitos en la educación artística (entre ellos juegos, danzas, y canciones), adquieren una preparación inicial previa a las pruebas de aptitud que deberán enfrentar. El continuar los estudios musicales especializados les ofrece una amplia formación en la que se integran conocimientos, competencias, valores, tecnología y demás, todo lo cual potencia su determinación hacia lo profesional, donde se puede ejercer en una gama de variantes entre ellas: la creación, la docencia, la dirección, la interpretación, la investigación, o la conjunción de algunas de ellas.

En México, son variadas las ofertas para optar por una licenciatura en alguna especialidad de música, existen más de treinta universidades que brindan al joven esta posibilidad (Universidades de México, 2020); ello impulsó esta reflexión que tiene por objetivo recapitular sobre aquellos aspectos que motivan a los estudiantes hacia la formación como músicos profesionales. 
Con base en lo expuesto por González (2008), un análisis motivacional contiene elementos de carácter afectivo (emociones y sentimientos), procesos cognoscitivos (sensopercepción, pensamiento, memoria, etc.) y de tendencias (voluntarias e impulsivas). Según el autor, los afectivos y las tendencias ocupan un lugar primordial, lo que indica la necesidad de su observación. Además, destaca el papel del objeto-meta del motivo como aspecto que distingue una actividad de otra.

\section{Desarrollo}

\section{Acerca de la motivación hacia el estudio}

En las publicaciones generales se puede encontrar diversas definiciones del término motivación. Algunas provienen de la década del cincuenta del siglo $\mathrm{XX}$, como resultado, se percibe una transición de significados que, aunque mantienen su esencia, se ajustan a las condiciones contextuales y la actividad que ejercen los diferentes autores. De esta forma, notamos su cualidad como condición, proceso, hecho, o constructo hipotético, entre otros. En las diferentes definiciones se sostienen aspectos referidos a la procedencia de las fuentes; al dinamismo, a la orientación, intensidad y persistencia. Valencia (2011) considera que las fuentes internas se relacionan con la historia genética, personal y las variables psicológicas de cada sujeto. Las externas, se refieren a diferentes estímulos que, aunque fuera del individuo, ejerce algún tipo de influencia sobre él. Otro elemento que menciona es el papel de la autorregulación, que "...puede predecir la persistencia en la tarea y se asocia positivamente con la regulación identificada, ..." (p.66).

Desde la posición de González (2008), la motivación incluye a los componentes cognoscitivo, afectivo y volitivo. Por ella entiende "...la compleja integración de procesos psíquicos que efectúa la regulación inductora del comportamiento, pues determina la dirección (hacia el objeto-meta buscado o el objeto evitado), la intensidad y el sentido (de aproximación o evitación) del comportamiento" (p.52). Este autor destaca la relación entre la motivación y el comportamiento; explicita que la primera lo inicia, mantiene, fortalece o debilita y finaliza una vez alcanzado el objetivo o meta. Igualmente, expresa cómo se revelan transformaciones o determinaciones en correspondencia con la influencia externa, lo cual se percibe como actividad motivada.
Por esta causa es necesario observar al estudiante durante el proceso formativo, ya que nos ofrece elementos que favorecen la elaboración de estrategias en aras de mantener o elevar la motivación hacia el estudio.

Con esta intención se precisa la necesidad de atender a las características del alumno que llega a nuestra clase porque decidió formarse como un músico profesional. Este viene con una carga de conocimientos adquiridos en niveles precedentes de escolaridad y en su entorno familiar, a la par de creencias y convicciones construidas como algo indudable, resultado de su experiencia, relaciones, reflexiones y emociones. Como profesores, debemos ser capaces de captar las señales que nos envía e identificar qué lo motiva, en qué nivel está, cómo es su preparación, cuánto interés muestra, qué música prefiere, cuáles son sus expectativas; es decir, observar patrones relativos fundamentalmente a la dirección o intensidad en su modo de actuar, así como en las respuestas afectivas en las actividades concernientes a su carrera. El alumno motivado se distingue en la clase por diferentes conductas: es activo, atiende las explicaciones, en ocasiones las cuestiona, se agota, ayuda, se adelanta a su turno, se interesa por su actuación, se ejercita fuera de la clase, entre otras (López y González, 2001).

Respecto a la motivación hacia el estudio, González (2004) precisa desde lo interno, el deseo de estudiar y aprender, lo que favorece la orientación hacia nuevos problemas cognitivos, principalmente durante la clase; de cualquier forma, recapitula la necesaria relación entre los motivos internos y los externos, así como el rol protagónico que tiene el docente y los métodos productivos que emplee. Según el autor, la motivación que estimula el deseo de avanzar en el estudio se compone por necesidades y motivos como elementos esenciales.

López y González (2001) exponen el papel de los motivos intrínsecos en el individuo respecto a una profesión, expresan que su formación es mucho más efectiva que la que se sustenta en los extrínsecos; asimismo plantean la relación entre el estado de satisfacción y la motivación manifiesta en las vivencias afectivas. 
Es decir, si se conoce y se tiene en cuenta lo que impulsa la acción del joven hacia un objetivo, el docente podrá accionar con mayor precisión sobre aquellos aspectos que influyan positivamente en el discípulo, lo que muestra la necesidad de fomentar una relación asertiva y comunicativa entre ambos, si es posible, durante toda su carrera; también se sugiere tomar en cuenta el entorno en que se desenvuelve el alumno. Si bien desde antaño "Conocer la música, cultivarla, denotaba una distinción del espíritu, una nobleza del saber, la insignia de la libertad..." (González-Cobo, 2016, p. 477); todavía hoy, en muchos contextos familiares y sociales de México, es importante estudiar una carrera que ofrezca "cierto nivel de reconocimiento social y económico", y en esta catalogación lamentablemente no están consideradas las artes y la música; ello constituye un motivo externo de impacto negativo.

Recapitulando, la motivación hacia el estudio es un proceso cuyas fuentes pueden ser internas o externas; regula la dirección e intensidad del estudiante hacia su meta, en este caso, relacionada con su preparación para el ejercicio de la música como profesión, y se revela, fundamentalmente, hacia la esfera afectiva.

\section{Motivación hacia el estudio de la música}

En el área de conocimiento de las artes, particularmente de la música, se pueden encontrar estudios referentes a este tema. Martínez (2018) los agrupa acorde a cuatro criterios:

- Motivación en el inicio de los estudios musicales; en este momento distingue la fuerza externa otorgándole a los padres, por lo general, un rol protagónico dada la influencia que ejerce sobre el niño a estas edades.
Motivación en el inicio de los estudios para ejecutar un instrumento musical; acá incluye aspectos de diversa procedencia, en algunos casos con un mismo patrón de estereotipos de géneros entre padres e hijos, al respecto podemos encontrar elementos discriminatorios de qué tipo de instrumento elegir si eres niña o niño, $u$ otros que tienen un impacto positivo como el de escoger el mismo instrumento que toca algún familiar cercano. También menciona las características del estudiante (edad, símbolos de identidad, persistencia, etc.), sociales (cultura, expectativas relacionadas con estereotipos, profesores, familiares, amigos) y las propias del instrumento.

Importancia de la motivación; en este aspecto destaca la relación entre el nivel alcanzado, el tiempo de estudio, las expectativas, el disfrute de la actividad, actitudes hacia la interpretación, autopercepción musical y empleo de estrategias para el estudio.

- Relevancia del contexto; considera la relación con otras personas, el apoyo, y la valoración sobre su estudio como elementos significativos.

En este último aspecto, donde se pone de manifiesto la importancia de las relaciones personales, incluso con la familia, en algunos artículos se hace visible la disminución del apoyo familiar con que se contaba al inicio de los estudios y el impacto que tiene en el cumplimiento de la meta del estudiante.

En una investigación realizada por Tripana (2010) el 52\% de los estudiantes encuestados consideraron un fracaso el hecho de no poder vivir de la profesión elegida, y declararon que les quedaba la satisfacción de haber estudiado lo que les gustaba, que pueden ser músicos y ganarse la vida con otro oficio. En él se revela que la mayor puntuación respecto a los factores de elección profesional como músicos, se asignó a la realización personal y a la estabilidad económica. 
En este último aspecto, es significativo cómo en nuestras aulas existen alumnos que constituyen las fuentes de ingreso en sus hogares, por lo que su tiempo de estudio disminuye; a esto se le adiciona que, al estar en un nivel superior, deben vencer otros programas que complementan su formación profesional.

Por otra parte, en los referentes teóricos se particulariza la necesaria continuidad de la influencia de los padres, al inicio, y de los docentes más tarde, de manera que se promueva el mantener el estudio de la música como eje fundamental de su formación, en aquellos que, además de las aptitudes necesarias, demuestren interés y presten especial atención a lo relacionado con una carrera que, desde la niñez, ha implicado asumir cierto grado de responsabilidad y sacrificio en la vida cotidiana de los niños: horas de juego, de relaciones sociales, o de distracción y descanso para dedicárselas, por ejemplo, a la práctica de un instrumento musical. Aunque se plantea que “...la elección de una profesión pone en juego la maduración de la personalidad y la adquisición relativa definida de una identidad" (Tripana, 2010), en el caso de la música, como ejercicio profesional, esta maduración se va consolidando tras años de estudios, ya que es una carrera que se selecciona desde edades tempranas.

En el nivel superior es el docente el encargado de orientar, enrumbar, estimular, y reforzar los motivos que llevaron al alumno a formarse como músico profesional; por ello, es menester una adecuada selección de los contenidos, dígase, por ejemplo, escalas, estudios, piezas y conciertos a interpretar, en armonía con una estrategia metodológica que combine la necesaria repetición práctica con la libertad de crear, recrear, enriquecer, y reflexionar, desde la propia música, teniendo en cuenta los objetivos, las habilidades técnicasexpresivas y competencias mínimas a desarrollar declarados en el programa para el nivel en que se encuentre el estudiante.

Otro aspecto a considerar es la orientación profesional como dinámica motivacional, dado que media en la elección, formación y desempeño profesional responsable. En este proceso, González (2013) apunta que intervienen la calidad del trabajo de los orientadores especialistas, agentes educativos de la escuela y la comunidad, y la familia, de acuerdo con los límites de la profesión de cada uno.
"En el caso de los profesores, ...la orientación profesional se realiza desde su rol de educadores en la medida que diseñan situaciones de aprendizaje desde sus asignaturas, la práctica investigativa o laboral potenciadoras de la motivación profesional" (González, 2013).

En las universidades mexicanas se ha implementado como complemento la actividad tutorial que tiene dos enfoques fundamentales, el primero de ellos, encauzado en la atención directa a estudiantes mediante el apoyo metodológico en su disciplina, y que va a tener en cuenta las dificultades en el aprendizaje de contenidos teóricos y prácticos; por otro lado, está la guía en el medio académico y universitario, donde el docente apoya al estudiante durante los años que dura su licenciatura. Casi siempre el maestro de la especialidad juega este rol de manera natural, pues lleva de continuo el desarrollo del estudiante, motivándolo para el logro de las metas, en atención a la culminación de los estudios profesionales.

\section{Metodología}

La estrategia metodológica usada combinó el análisis de documentos, la entrevista no estructurada y la observación pedagógica para identificar los motivos por los que los alumnos deciden estudiar la música como carrera, declarar esferas en las que esta impacta, y concretar acciones para el fomento de la motivación en los estudiantes. El resultado se obtuvo a partir de una triangulación metodológica que facilitó el análisis de datos, la comparación y discriminación de información. Con estos fines se tomó una muestra casos tipo (Hernández, Fernández, y Baptista, 2014); se entrevistaron 10 maestros de música y 15 alumnos que cursan la licenciatura en algún instrumento musical.

Para el análisis de documentos se revisó un total de 78 fuentes (tesis doctorales y de maestría, artículos de revistas indizadas y capítulos de libros) que referían el estudio de la música; el $87 \%$ del presente siglo XXI, y de ellos, el $73.5 \%$ de la última década. Como unidades de análisis se tomaron aquellas fuentes bibliográficas que refirieran la motivación para el estudio de la música y el impacto de esta en la sociedad; ello redujo la muestra a 42 documentos que enuncian ideas sobre el impacto de la música y 23 con temas relacionados con su estudio como carrera.

JUAN-CARVAJAL, Mara Lioba, JUAN-CARVAJAL, Dargen Tania y VDOVINA, María. La motivación en la música como eje de la formación profesional. Revista de Gestión Universitaria. 2020 
En cuanto a las categorías de análisis se adaptaron aquellos aspectos en los que se expresa la motivación propuestos por González (2001) agrupadas en dos dimensiones: direccional, las que se orientaban al acercamiento del estudio por la carrera; de activación, las que muestran la intensidad con que el estudiante se acerca a su objetivo; lo cual posibilitó la determinación de los motivos y su jerarquización.

La observación pedagógica y la entrevista ofrecieron información sobre la motivación de los alumnos hacia el estudio. Al concluirla se empleó la técnica Tres ideas, se le entregó una hoja al entrevistado y se le solicitó que escribieran en ella tres (3) ideas sobre los motivos para estudiar música (para el caso de los alumnos); a los docentes se les pidió igual, pero respecto a sus estudiantes. También se les invitó a que escribieran otras tres sobre si consideran la música importante para la sociedad y para qué. Estas se revisaron, agruparon y ordenaron jerárquicamente de acuerdo con la cantidad de repeticiones; se determinó la media y se escogieron aquellas cuyo valor era igual o superior a 12 para el caso del estudio y 14 para el impacto.

\section{Resultados}

De la triangulación metodológica realizada se apreció que, aunque los datos fueron obtenidos por diferentes vías, existía cierta similitud en la información manifiesta entre la teoría signada tras la experiencia de docentes y psicólogos que investigaron sobre el tema de la motivación del estudio hacia la música, y el criterio de profesores y alumnos de esta rama del arte, en la universidad.

Desde el punto de vista de los estudiantes y docentes se identificaron esferas en las que el ejercicio de la música influía en la calidad de las actividades; entre ellas se distinguen los proyectos personales, las trasformaciones sociales a través de proyectos (social, educativo, cultural, comunitario); mejora en la capacidad de memorizar, en el rendimiento académico, en las respuestas emocionales; desarrollo de valores y fomento de relaciones, desarrollo de capacidades críticas y de apreciación de las artes, y, en la mayoría de los casos, mencionaron el descanso y la diversión.
En lo referente a las motivaciones para el estudio de la música, la información se concentra en:

\section{- $\quad$ El reconocimiento social \\ - $\quad$ La influencia del entorno en que viven \\ - Realización y satisfacción personal \\ - Alcanzar mayor cultura \\ - Búsqueda de una salida profesional \\ - Pertenecer a una banda o grupo musical \\ - Tocar un instrumento musical ya sea como solista, en agrupaciones y orquestas, o ambas \\ - Disfrutar la música y tener la oportunidad de hacer que otros se recreen con ella \\ - Demostrar su habilidad en grupos \\ - Tocar en iglesias, eventos religiosos o sociales \\ - $\quad$ Necesidad de expresarse y comunicarse a través del arte}

En la entrevista con los docentes se puso de manifiesto la observancia de la actuación de los alumnos; se reiteró la atención prestada, los buenos resultados académicos con carácter sistemático, la satisfacción expresa por los estudiantes por recibir clases con esos profesores y por la elevación del prestigio ante sus compañeros. En sentido negativo, se descubre la insatisfacción por la falta de tiempo para el estudio debido a que en este nivel existen alumnos que trabajan y estudian, e incluso tienen familias a su cargo. También tienen que hacer gastos relacionados con la renta, transporte y alimentación, ya que no todas las universidades tienen como oferta la carrera de la música. En muchas ocasiones están obligados por la familia a terminar una carrera como el derecho, la ingeniería u otra, para entonces poder concluir los estudios de la música como profesión.

Como resultado de la técnica Tres ideas, se concretaron acciones para mantener o reforzar la motivación hacia el estudio de los alumnos de música. En ella se destacó la necesidad de dirigir la atención, en primer lugar, hacia las acciones relacionadas con los modos de actuación profesional, al diseño didáctico de las clases y a la observancia del comportamiento. 
Las acciones expresadas se centran en reconocer la orientación profesional como eje transversal en la carrera; el incremento de actividades prácticas donde se propicie y estimule el debate y la reflexión sobre interpretaciones magistrales, el empleo de la tecnología, el debate y la reflexión mediante la participación en cursos, foros, festivales, actividades de conciertos y eventos extracurriculares que expliciten las amplias posibilidades de desarrollo, escuchando y reconociendo otras experiencias de artistas nacionales e internacionales; la audición de obras de importancia relevante por la calidad de su interpretación o, los elementos compositivos y valores estéticos de diferentes épocas; la movilización e intercambio estudiantil por semestres o períodos cortos hacia otras universidades con tipologías similares, las actividades de conciertos que posibilita la experiencia cultural educativa y la vinculación social; la caracterización del estudiante y su correspondencia por asumir la responsabilidad en su formación; dinamizar el rol del docente de acuerdo con el entorno en que se desarrolla el proceso; y la cooperación, así como la asistencia al estudiante para fijar sus metas.

\section{Conclusiones}

La música ha acompañado a las sociedades en los procesos evolutivos del desarrollo de la humanidad, y todavía continúa ocupando un lugar cimero en el contexto socio-cultural y el progreso de los países. El arte musical influye en el hombre tanto en la esfera afectiva como en las cognitiva y volitiva; de esta manera, en los diferentes niveles del sistema educativo en México, se observan transformaciones metodológicas en los programas de educación artística que pretenden aprovechar este impacto a favor del desarrollo del niño y el adolescente.

Existe una relación entre la motivación y la intensidad y dirección del comportamiento que se revela como patrones en los modos de actuación de los estudiantes. La observancia de estos patrones favorece al docente el diseño de acciones que impacten positiva y sistemáticamente, en la motivación hacia el estudio de la música.
La correspondencia en las respuestas resultantes de la aplicación de los métodos y técnicas facilitó la identificación de los motivos que impulsan a los estudiantes a adoptar la música como eje de su formación profesional, en este sentido, se apreció la existencia de motivos extrínsecos e intrínsecos, los más reiterados resultaron el reconocimiento social y la realización personal.

\section{Referencias}

Aróstegui, J.L.; Louro, A.L.; y Lima, Z. (2015). Las políticas educativas de reforma y su impacto en la Educación Musical Escolar. De dónde venimos y hacia dónde podemos ir. Revista da ABEM 23 (35), 24-34.

Carrillo, C.; Viladot, L.; Pérez-Moreno, J. (2017). Impacto en la educación musical: una revisión de la literatura científica, en Revista Electrónica Complutense de Investigación en Educación Musical (14), 61-74.

Conejo, P.A. (2012). El valor formativo de la música para la educación en valores. DEDiCA. Revista de Educação e Humanidades (2), 263278.

Cremaschi, A. (2011). Identidad motivación y objetivos en alumnos mexicanos de piano clásico: un análisis preliminar. En UJAT-CEDA ISME 2011. Ira Conferencia Regional Panamericana. Conferencia llevada a cabo en 8 va Conferencia Latinoamericana. Recuperado de

https://www.researchgate.net/publication/31507 4926_IDENTIDAD_MOTIVACIÓN_Y_OBJE TIVOS_EN_ALUMNOS_MEXICANOS_DE_ PIANO_CLASICO_UN_ANALISIS_PRELIM INAR.

González, A. (2004). La motivación hacia el estudio. Fundamentos y metodología para su evaluación en secundaria básica (tesis doctoral). Ministerio de Educación. Cuba.

González, D. (2001). Teoría de la motivación y práctica profesional. La Habana: Ed. Pueblo y Educación.

González, D. (2008). Psicología de la motivación. La Habana: editorial Ecimed. 
González, V. (2013). La orientación profesional en la educación superior. Reflexiones y experiencias desde el enfoque histórico-cultural del desarrollo humano. Alternativas cubanas en psicología 1(2). Recuperado de http://acupsi.org/articulo/30/la-orientacionprofesional-en-la-educacion-superior\%2520refelxiones-y-experiencias-desde-el-enfoquehistorico-cultural-del-desarrollo-humano.html

González, V. et al. (2000). Psicología para educadores. La Habana: Ed. Pueblo y Educación.

González-Cobo, R.A (2016). El mundo en el oído. El nacimiento de la música en la cultura.

Barcelona: Acantilado. (2014). Diccionario de música, mitología, magia y religión. Barcelona: Acantilado.

González-Moreno, P. (2013). Motivación estudiantil hacia el estudio de la música: el contexto mexicano. Revista Internacional de Educación Musical RIEM (1), 31-41.

Hernández, R.; Fernández, C.; y Baptista, P. (2014). Metodología de la Investigación. México: McGraw-Hill / Interamericana editores, S.A. de C.V.

López, A; y González, V. (2001). Niveles de satisfacción por la clase de Educación Física. Efdeportes.com Revista digital-Buenos Aires (32). Recuperado de https://www.efdeportes.com/efd32/satisf.htm

Lorenzo, M. (2020). Las escuelas de música y los proyectos musicales socioeducativos y comunitarios. Modelos educativos, retos y contextos. ArtsEduca (27), 36-53.

Martínez, I. (2018). Motivación y emoción en la música. Estado de la cuestión y aportaciones para la innovación educativa. Educación y Pedagogía (19), 63-82.

Miranda, M.; Hazard, S.; y Miranda, P. (2017). La Música como herramienta terapéutica en medicina. Rev Chil Neuro-Psiquiat 55 (4), 266277.

Morales, M. E. (2010). La Escuela de Música de la Universidad de Guadalajara, 1952-2004: preludio y desarrollo de una institución jalisciense de la música académica. México: Universidad de Guadalajara. Centro Universitario de Arte, Arquitectura y Diseño.
Piñeros, G. (2016). Influencia de la música en el proceso de enseñanza-aprendizaje en estudiantes de medicina (tesis doctoral) Universidad de Granada, Colombia.

Rueda, Y.M. (2020). Incidencia de la música en la transformación social y la construcción de paz en el territorio rural de Catambuco (NariñoColombia). RICER CARE (13), 26-55.

Tripana, S. (2010) Motivación y orientación profesional en los estudiantes de música. REME. Revista Electrónica de Motivación y Emoción XIII (34), Disponible en http://reme.uji.es/articulos/numero34/article3/te xto.html

Universidades de México (2020). Carreras y licenciaturas en Licenciatura en Música. México. Recuperado de https://universidadesdemexico.mx/carreras/lice nciatura-en-musica.

Valencia, R. (2011). Motivación académica en alumnos del Conservatorio Profesional de Música de Las Palmas de Gran Canaria y del Conservatorio Superior de Música de Canarias (tesis doctoral). Universidad de las Palmas de Gran Canaria. España. 